\title{
Um Ambiente Virtual Multijogador como Ferramenta Auxiliar para o Ensino de Estudantes de Medicina
}

\author{
Rodrigo Monteiro de Lima², Araken de Medeiros Santos², Francisco Milton \\ Mendes Neto ${ }^{2}$, Francisco Tailânio de Macedo ${ }^{1}$, Ademar França de Sousa Neto ${ }^{1}$, \\ Felipe César Pinheiro Leão ${ }^{1}$, Maria Luzia Silva de Carvalho ${ }^{1}$, Fernando Henrique \\ Alves $^{1}$ \\ ${ }^{1}$ Bacharelado em Ciência da Computação - Departamento de Ciências Exatas e Naturais \\ - Universidade Federal Rural do Semiárido (UFERSA) - BR 110 - Km 47 - 59625-900$$
\text { - Mossoró - RN - Brasil. }
$$ \\ ${ }^{2}$ Programa de Pós-Graduação em Ciência da Computação - Departamento de Ciências \\ Exatas e Naturais -Universidade Federal Rural do Semiárido (UFERSA) - BR 110 - \\ Km 47 - 59625-900 - Mossoró - RN - Brasil. \\ rodrigomonteiro89@hotmail.com, \{araken,miltonmendes\}@ufersa.edu.br, \\ tailanio@outlook.com, ademarnetol4@gmail.com, felipecpl11@gmail.com, \\ luziaf2@hotmail.com, fernando_fha0l@hotmail.com
}

\begin{abstract}
This paper describes a multiplayer virtual environment as an aid in the teaching and learning of medical students. The $3 D$ environment is composed of NPCs (Non Playable Characters) that interact with users in order to offer some sort of educational content. Due to be multiplayer, students can communicate within the environment with other students and share knowledge. Users can have two profiles: student and professor. Professors can see which areas their students have more difficulty. Students, besides interacting with the environment, can analyze virtual patients through simulation of clinical cases. By achieving goals, the student is rewarded with achievements.
\end{abstract}

Resumo. Este artigo descreve um ambiente virtual multijogador como auxílio no processo de ensino e aprendizagem de estudantes de medicina. $O$ ambiente $3 D$ é composto de NPCs (Non Playable Characters) que interagem com os usuários, a fim de ofertar algum tipo de conteúdo educacional. Por ser multijogador, estudantes podem se comunicar dentro do ambiente com outros alunos e trocar conhecimentos. Usuários podem ter dois perfis: estudante e professor. Professores podem visualizar quais áreas seus alunos possuem mais dificuldade. Os estudantes, além de interagir com o ambiente, podem analisar pacientes virtuais através da simulação de casos clínicos. Ao atingir metas, o estudante é recompensado com conquistas.

\section{Introdução}

Para Lima (2012), professores utilizam recursos adicionais para simular fenômenos que estão sendo estudados em sala de aula. Porém, essa prática limita-se apenas à demonstração de fenômenos apresentados pelo professor. A participação do aluno continua sendo de expectador e o mesmo não tem a oportunidade de realizar experimentos ou simulações. A causa disso pode ser a falta de recursos da instituição de 
ensino, como recursos financeiros e espaços disponíveis ou inviabilidade devido a riscos ambientais ou mortais. A realidade virtual se encaixa em situações desse tipo, onde a falta de recursos e riscos existem, pois a mesma não trás riscos a nenhum meio, visto que tudo é simulado por computador [Brasil 2011].

Dados do Superior Tribunal de Justiça do Brasil apontam para um aumento no número de denúncias por erros médicos a partir de 2011. No mesmo ano, o Conselho Federal de Medicina decidiu realizar um teste não obrigatório com estudantes do último semestre do curso de medicina por todo o país, a fim de verificar os conhecimentos adquiridos pelos estudantes no decorrer do curso. Os resultados foram preocupantes, pois $46 \%$ dos estudantes foram reprovados. Os estudantes apresentaram desconhecimento no diagnóstico e tratamento para infecção de garganta, meningite, sífilis, entre outras doenças [G1 2012].

O teste passou a ser obrigatório apenas no estado de São Paulo, no ano de 2012, pelo CREMESP (Conselho Regional de Medicina de São Paulo). O resultado do exame no estado, nesse ano, teve um índice de $54,38 \%$ de aprovação por estudantes da rede particular de ensino e $63,7 \%$ de aprovação por estudantes de universidades públicas. O índice de reprovados nos anos de 2013 e 2014 foi de, respectivamente, $54,5 \%$ e $59 \%$ [CREMESP 2014].

Este artigo está organizado da seguinte forma: na Seção 2, são apresentados alguns trabalhos relacionados à pesquisa. $\mathrm{Na}$ Seção 3, são explicados conceitos de gamificação. A Seção 4 apresenta a arquitetura do sistema, detalhes de desenvolvimento e algumas interfaces da aplicação. Por fim, a Seção 5 traz as considerações finais e perspectivas de trabalhos futuros.

\section{Trabalhos relacionados}

Kera, Pedrini e Nunes (2011) afirmam que o treinamento de procedimentos médicos pode ser beneficiado com o uso de ambientes virtuais interativos que simulam com realismo as ações do usuário. A simulação deve emitir respostas rápidas relativas ao encontro de objetos, deformação, restrição de movimento ou mesmo produzir forças e vibrações.

O ambiente desenvolvido visa à simulação do procedimento de punção, o qual consiste na extração de pequenas partes de tecidos do órgão em questão com o objetivo de auxiliar a elaboração do diagnóstico médico. Além da criação do ambiente, os autores criaram uma metodologia para criação de um ambiente virtual para treinamento médico com enfoque na colisão e deformação de objetos do ambiente virtual. A Figura 1 mostra a colisão entre uma seringa e uma mama. 
CBIE-LACLO 2015

Anais dos Workshops do IV Congresso Brasileiro de Informática na Educação (CBIE 2015)

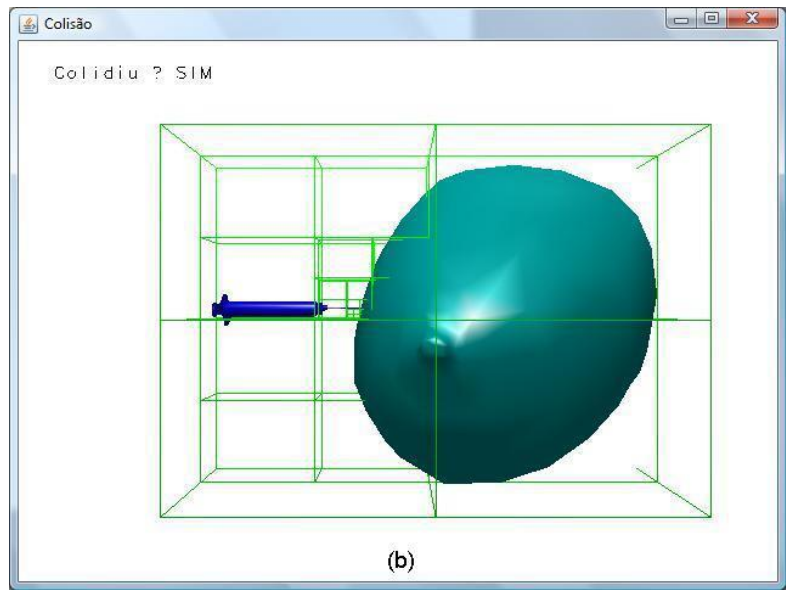

Figura 1. Experimentos entre colisões de objetos 3D entre uma seringa e uma mama [Kera 2011]

Pesquisadores do Rio Grande do Sul, no Brasil, usaram técnicas de inteligência artificial para criação de um simulador de casos clínicos. O sistema possui um sistema multiagente composto por três agentes: agente de domínio, agente de aprendizagem e agente mediador. Intitulado de SimDeCS, a ferramenta segue o processo médico do ensino técnico e especialização baseando-se em consultas médicas, atendimento, aulas e sessões de discussão. O estudante de medicina pode utilizar a ferramenta para desenvolvimento de suas habilidades e competências sobre diagnósticos formulados [Bez 2014].

Frade et. al. (2014) desenvolveram o UniVirtual, um ambiente virtual 3D multiagente com recomendação personalizada de objetos de aprendizagem. Devido aos avanços dos recursos tecnológicos, novas possibilidades surgiram nos processos de ensino e aprendizagem. No entanto, mesmo com a melhoria nos processos da Educação a Distância (EaD), a evasão ainda continua sendo um grande problema enfrentado pelas instituições de ensino. Em vista disso, os pesquisadores criaram um ambiente 3D com o objetivo de aumentar a motivação dos alunos em estudar, mesmo após as aulas em sala de aula. A Figura 2 mostra a arquitetura do sistema proposto.

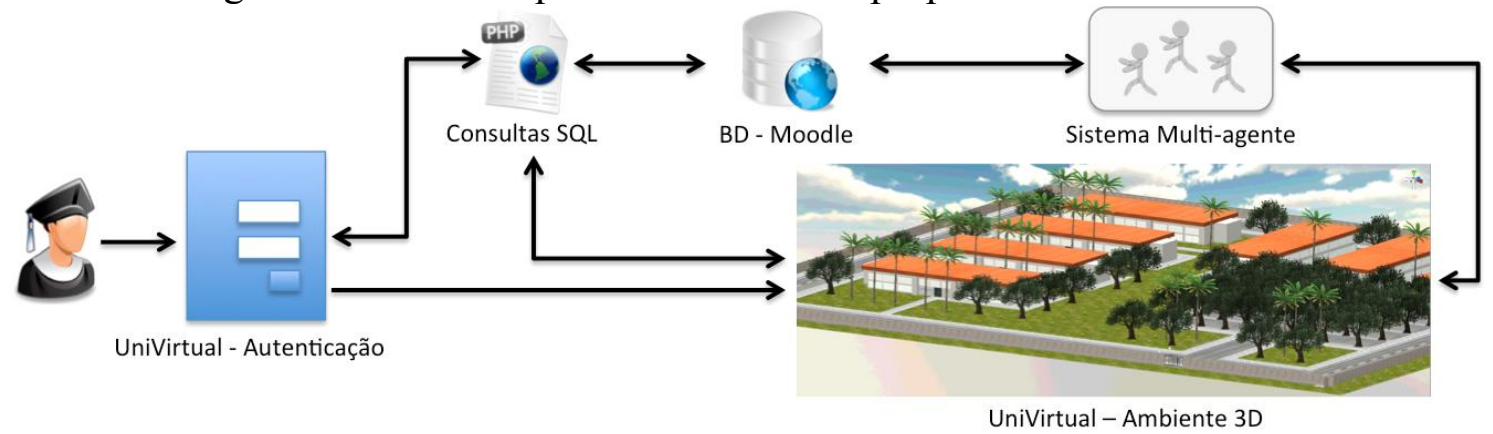

Figura 2. Arquitetura da UniVirtual [Frade 2014]

Veras et al. (2013) criaram um ambiente para treinamento e diagnóstico aplicado à mastologia usando inteligência artificial. Os autores usaram sistemas multiagente, redes neurais, ontologia e transformada de Fourier para criação do ambiente. O objetivo da ferramenta é o treinamento de profissionais na área da saúde através de e-learning e diagnóstico de doenças que afetam a glândula mamária. De acordo com os autores, o número de pacientes com problemas relacionados às glândulas mamárias está crescendo no Brasil. 
Mediante os trabalhos apresentados, não se encontrou nenhum trabalho que utilizasse ambientes 3D para o ensino e simulação de casos clínicos multijogador e multiplataforma. Em vista disso, este artigo propõe um ambiente com aspectos de gamificação, onde o aluno pode treinar conceitos de medicina em um ambiente realista e divertido e o professor pode analisar em quais pontos seus alunos possuem mais dificuldade para assim sanar as dúvidas virtualmente ou presencialmente.

\section{Gamificação}

De acordo com Hamari, Koivisto e Sarsa (2014), durante os últimos anos a gamification, ou gamificação em português, tem sido tendência em novos estudos como forma de apoiar o envolvimento dos usuários e melhorar a sua experiência e desempenho em qualquer atividade. Alguns dos objetivos da utilização dessa tecnologia em trabalhos são: aumentar a atividade do usuário junto ao sistema e aumentar a interação social e a qualidade produtiva de suas ações.

É estimado que mais de $50 \%$ dos processos de inovações nas empresas irão inserir aspectos de gamificação em seus negócios até 2015. Além disso, existe um número crescente de startups focadas em adicionar uma camada com características de jogos, como é o caso da codecademy, que é um serviço que usa elementos de jogos com o objetivo de facilitar o ensino de programação para estudantes [Sims 2015].

$\mathrm{O}$ interesse em gamification também é refletido na área acadêmica. O número de artigos publicados sobre o tema está crescendo, como mostra a Figura 3. O papel da gamificação de processos tem como objetivo trazer as mesmas experiências psicológicas que os jogos trazem para atividades que não são jogos propriamente ditos. Por outro lado, outros pesquisadores afirmam que o termo tem como objetivo trazer as mesmas características explícitas usadas em jogos, independentemente dos resultados obtidos [Hamari 2014].

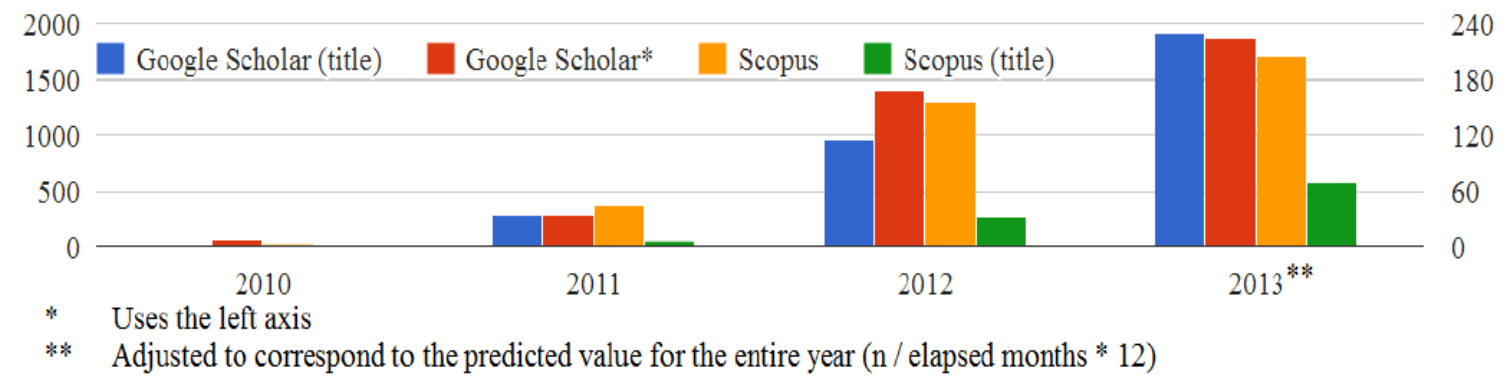

Figura 3. Resultados sobre a utilização de gamificação em trabalhos científicos entre os anos de 2010 e 2013 [Hamari 2014]

\section{Um Ambiente Virtual para o Ensino de Conceitos de Medicina}

Em vista da quantidade de erros médicos nos últimos anos, juntamente com a motivação de criar um sistema onde estudantes de medicina pudessem aprender conceitos da área da saúde e treinar diagnósticos com pacientes virtuais, foi criado esse mundo virtual. Nesta seção serão apresentadas as principais características do sistema. A Subseção 4.1 mostra a arquitetura do ambiente. A Subseção 4.2 apresenta os aspectos de implementação do ambiente proposto. A Subseção 4.3 mostra interfaces do sistema e um cenário de uso. 
CBIE-LACLO 2015

Anais dos Workshops do IV Congresso Brasileiro de Informática na Educação (CBIE 2015)

\subsection{Arquitetura Física}

O ambiente virtual pode ser executado em diversos dispositivos computacionais, tais como: computadores de mesa, notebooks, tablets e smartphones. Através de conexão de internet, os usuários podem entrar e interagir com o mundo virtual. $\mathrm{O}$ ambiente virtual precisa estar conectado à Internet, pois precisa haver comunicação com diversos serviços na nuvem. A Figura 4 detalha a arquitetura do sistema.

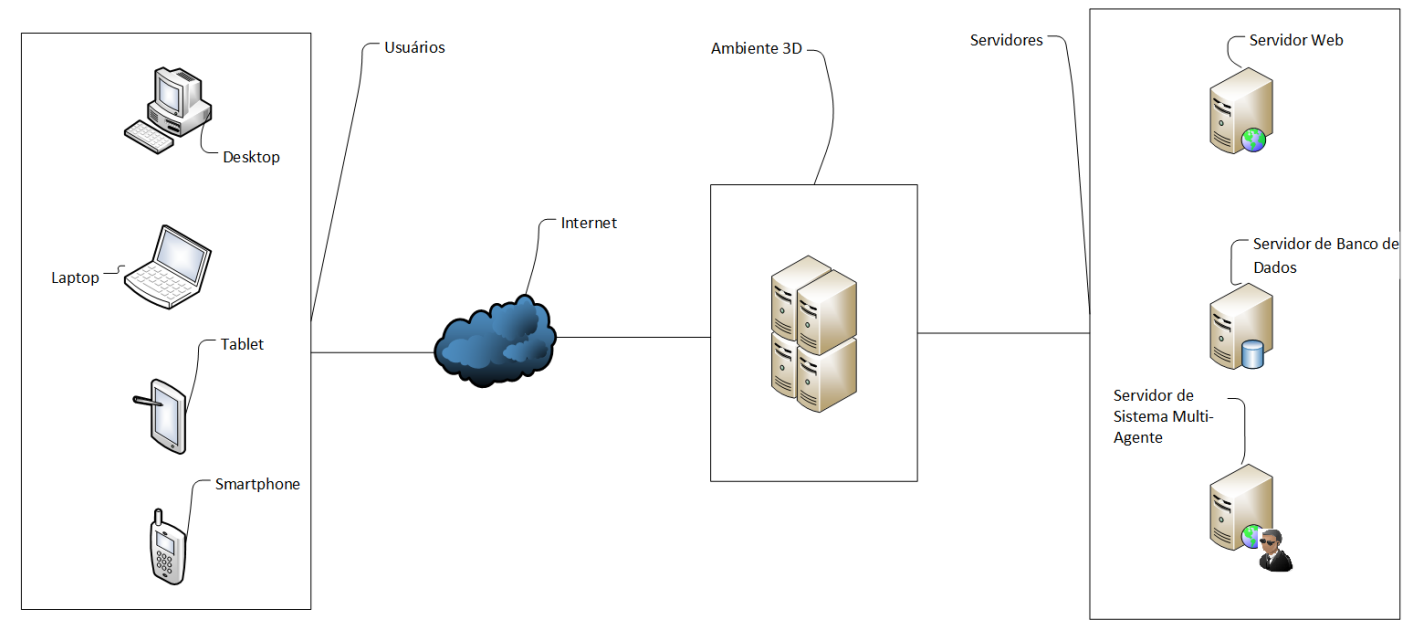

Figura 4. Arquitetura do sistema

\subsection{Aspectos de Implementação}

O ambiente virtual foi desenvolvido dentro de um mundo virtual já existente, desenvolvido pelo grupo de pesquisa dos autores, chamado de UniVirtual [Frade 2014]. A ideia de utilizar um ambiente virtual já existente se deu pelo fato da prévia validação desse ambiente, bem como no ganho de tempo de se utilizar um arcabouço já desenvolvido e validado no próprio grupo de pesquisa dos autores. O UniVirtual tem como base a ideia de unir várias áreas de conhecimento oferecendo um espaço virtual onde os alunos possam ser levados para mundos virtuais a partir de suas áreas de interesse. Ao entrar no ambiente, o estudante pode ir para outros mundos virtuais, entrando em áreas específicas. O ambiente é livre, bastando apenas entrar em contato com os desenvolvedores para a inserção de novos mundos virtuais.

Para criação dos objetos 3D presentes no ambiente virtual, foi utilizado o programa Blender [Roosendaal 2002]. Para manipulação e carregamento de modelos em três dimensões, foi utilizado um motor gráfico presente na ferramenta Unity $3 D$ [Unity 2015]. Essa IDE (Integrated Development Environment) possui uma versão gratuita com diversas funções para criação e gerenciamento de ambientes virtuais $2 \mathrm{D}$ ou $3 \mathrm{D}$. $\mathrm{O}$ editor possui uma variedade de funções que podem ser usadas para criação de animações, jogos ou simuladores. A IDE também conta com uma versão paga mais robusta e com mais funcionalidades. A programação do ambiente virtual foi realizada via drag and drop (arrastar e largar), para disposição dos objetos 3D presentes no ambiente, e através das linguagens de programação C\# [Jennifer 2010] e JavaScript [Silva 2010], para configuração de funções como multijogador, multiplataforma, chat, entre outras. 


\subsection{Cenário de Uso e Interfaces do Ambiente Virtual}

Primeiramente, o estudante precisará efetuar o cadastro no sistema. Ao entrar no site de divulgação do trabalho (http://www.les.ufersa.edu.br/doctraining), o aluno pode se autenticar no sistema de cadastro, inserindo seus dados. Após a inscrição ser efetuada com sucesso, ele pode utilizar suas credencias de entrada, login e senha, para entrar no mundo virtual.

Após entrar pela primeira vez, o sistema pergunta se o usuário deseja um personagem feminino ou masculino. Essa pergunta se dá pelo fato de que uma pesquisa realizada com 375 jogadores na Syracuse University, nos Estados Unidos, apontou que $25 \%$ desses jogadores preferiam jogar com personagens femininos [Marcial 2014]. Os entrevistados afirmam que dessa maneira podem ser mais emotivos e não sofrer preconceito. Em vista disso, pensou-se em manter essa opção no sistema. A Figura 5 apresenta a tela de escolha de personagens do ambiente.

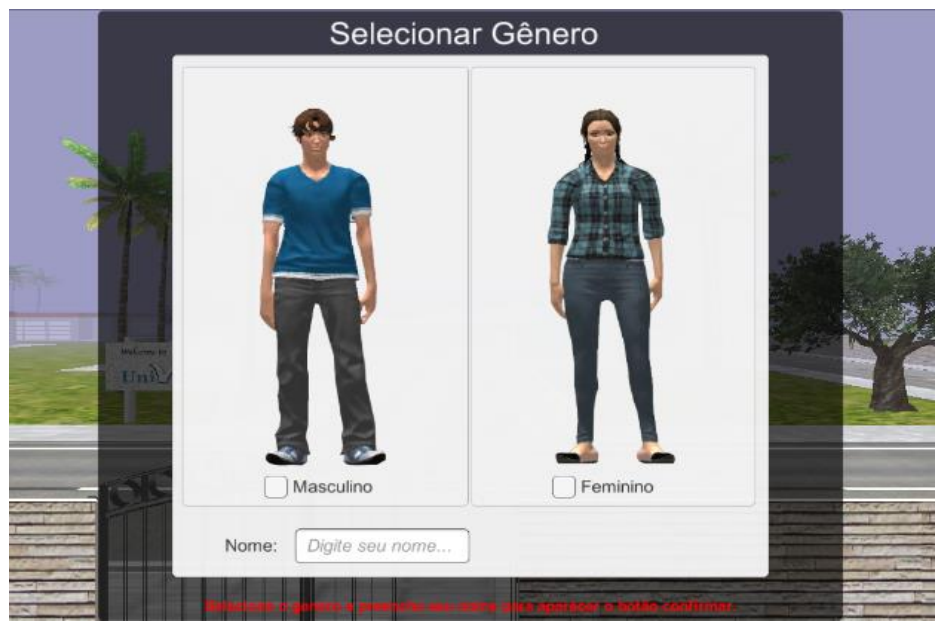

Figura 5. Tela de seleção de gênero de personagem

Dentro do ambiente, jogadores podem interagir com NPCs. Esses personagens controlados por computador oferecem conteúdos da área de medicina aos alunos. Alguns oferecem também perguntas aos jogadores, a fim de fazer com que o estudante esteja sempre ativo no processo de aprendizagem e não apenas passivo. Cada NPC possui diálogos sobre diversos assuntos, como pediatria, cirurgias, doenças, sintomas, vírus, materiais cirúrgicos, entre outros. A Figura 6 (a) mostra o usuário se aproximando de um NPC e a opção de conversa sendo apresentada. A Figura 6 (b) mostra o NPC oferecendo algum conteúdo para o estudante. 


\section{CBIE-LACLO 2015}

Anais dos Workshops do IV Congresso Brasileiro de Informática na Educação (CBIE 2015)

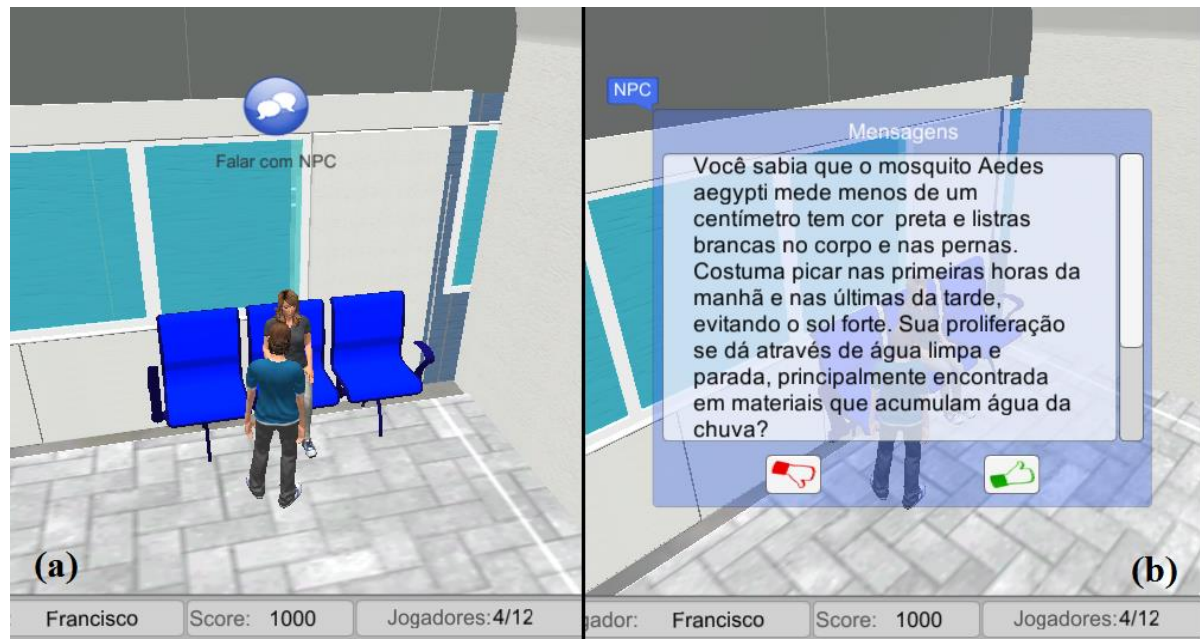
Figura 6. (a) Jogador se aproximando de NPC (b) Conteúdo educativo passado
para o aluno

Como o ambiente é multijogador, é comum que estudantes se encontrem com outros estudantes. Quando isso acontece, jogadores podem interagir com outros alunos através de chat. Para isso, deve-se ativar a opção de conversa para que a janela de bate papo fique ativa. Feito isso, basta digitar mensagens para os outros estudantes.

A principal motivação para o uso dessa característica é a interação entre jogadores sobre assuntos educativos. Dessa maneira, o aluno pode trocar informações com outros estudantes sobre dificuldades que o mesmo encontrou ou novos conhecimentos adquiridos no ambiente. A Figura 7 mostra a interação entre dois jogadores no mesmo ambiente.

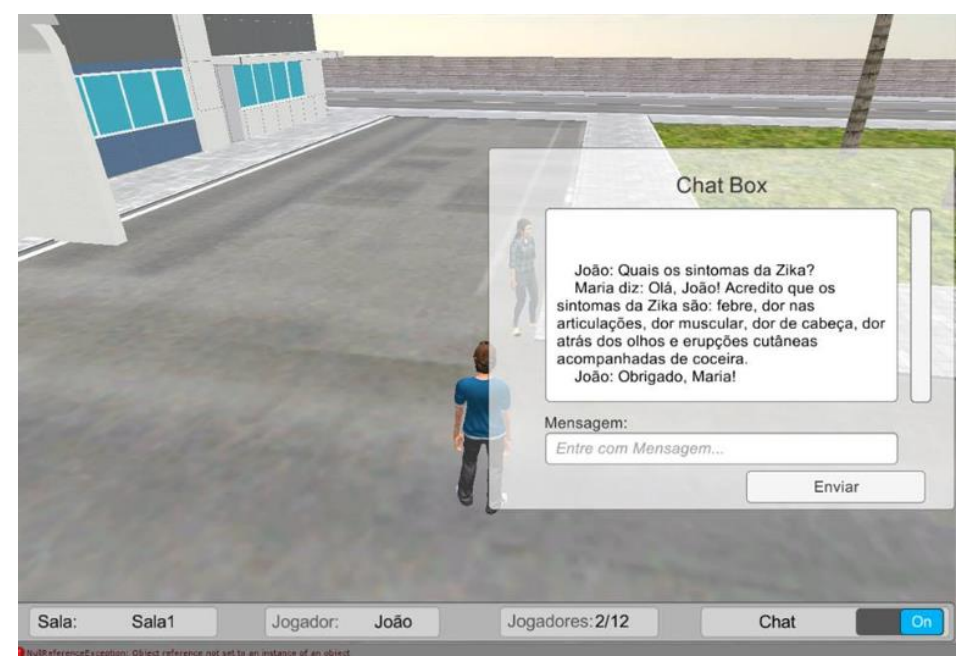

Figura 7. Interação entre dois jogadores

Além de assuntos ofertados pelo ambiente 3D, jogadores podem consultar pacientes através de consultórios virtuais. Após entrar no hospital universitário, o estudante pode entrar em seu consultório médico, a fim de avaliar os pacientes na lista de espera. Quando um paciente entra na sala de consulta, é iniciado um diálogo entre paciente e estudante. $\mathrm{O}$ paciente fala para o médico quais sintomas o mesmo está apresentando. 


\section{CBIE-LACLO 2015}

Anais dos Workshops do IV Congresso Brasileiro de Informática na Educação (CBIE 2015)

A partir desse momento, o aluno pode entrar com um diagnóstico ou pedir para o paciente ir para a sala de exames. Dentro da sala de exames, o médico, controlado pelo jogador, pode examinar sintomas no corpo do paciente que não foram informados anteriormente. Esses sintomas podem ser manchas no corpo, lesões, entre outros. A Figura 8 mostra o paciente conversando com o médico e falando quais sintomas está sentindo.

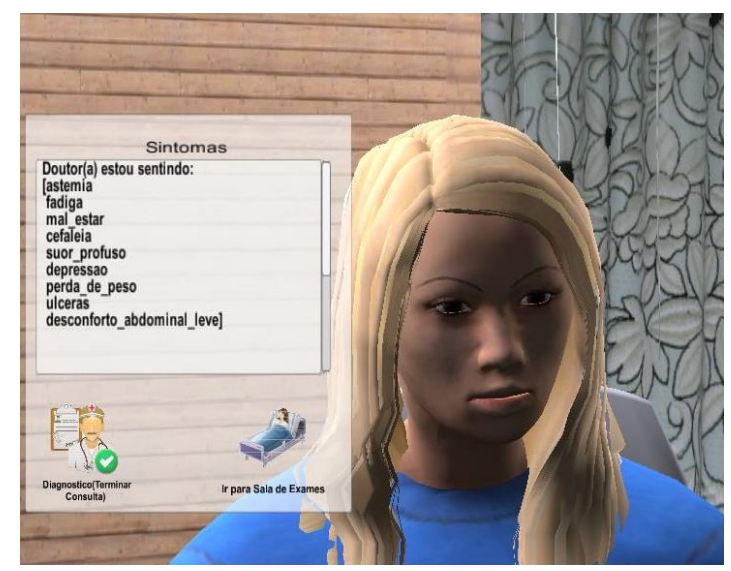

Figura 8. Paciente dizendo os sintomas que está sentindo ao jogador

Outra característica presente no sistema são as conquistas (badges). Uma badge ou conquista é uma credencial digital que representa habilidades, interesses e realizações individuais [Mozilla 2013]. Ao analisar a literatura acadêmica, estudos de casos, relatórios de projetos e experiências pessoais relatadas por pesquisadores e profissionais da educação, constatou-se que o mecanismo motivacional, o método de reconhecimento e certificação e os indicadores de aprendizagem são os principais papéis das conquistas no ambiente educacional [Jovanovic 2014]. Conquistas são metas estipuladas pelos administradores do sistema e professores. Cada conquista possui nome e descrição. Professores podem criar suas próprias conquistas a partir do sistema. Após preencher os dados obrigatórios das conquistas, os administradores do sistema precisam verificar se as mesmas são válidas para uso no ambiente virtual. Depois da validação, as conquistas ficam disponíveis para qualquer usuário do sistema. A Figura 9 apresenta um estudante obtendo uma conquista após interagir com mais de 15 NPCs presentes no mundo virtual.

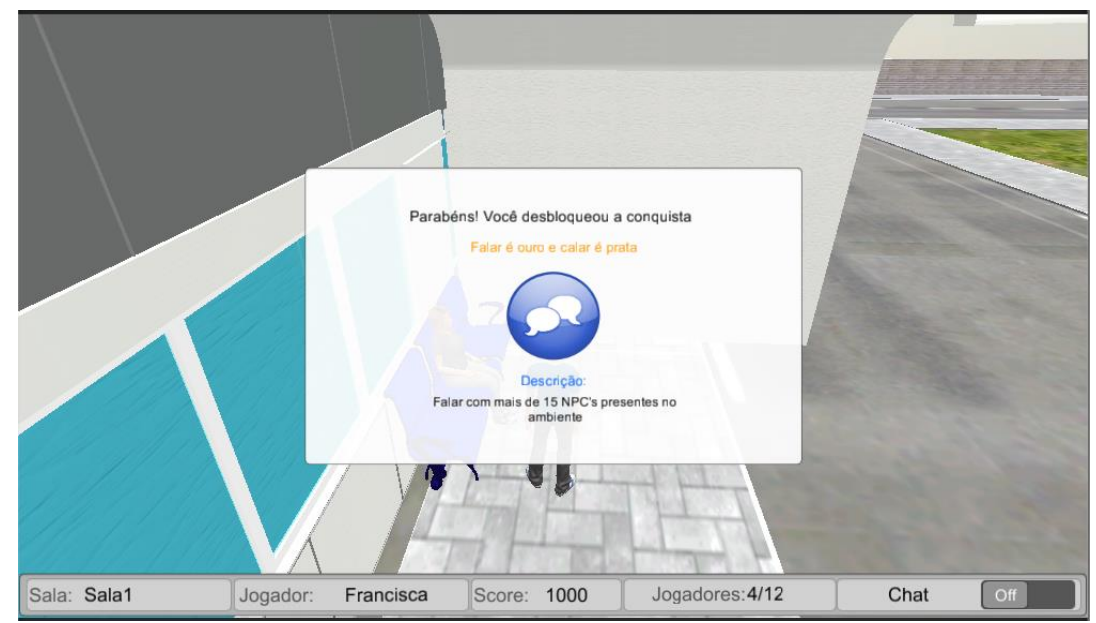

Figura 9. Obtenção da conquista "Falar é ouro e calar é prata" 


\section{Conclusões e Trabalhos Futuros}

A partir de estudos realizados sobre trabalhos relacionados nesta área, não foi encontrado nenhum ambiente em três dimensões capaz de oferecer um ambiente com características de gamificação, NPCs que passem conteúdos na área médica, simulação de consultas e uso de conquistas como mecanismo motivador para a utilização do sistema. O principal objetivo da ferramenta é oferecer uma ferramenta gratuita para as universidades, onde estudantes de medicina possam treinar casos clínicos simulados, bem como receber conteúdos educacionais fora da sala de aula. A utilização de um ambiente virtual 3D teve como propósito proporcionar um ambiente realista e intuitivo. Por estar disponível na nuvem e ser multiplataforma, o ambiente virtual pode ser executado a qualquer instante e em qualquer dispositivo computacional.

Como trabalhos futuros, espera-se criar uma comunicação simulada entre pacientes virtuais e alunos de medicina através de dispositivos móveis. O objetivo é usar o sintetizador de voz presente em alguns smartphones para simular uma ligação entre paciente e médico, simulando situações adversas. Por exemplo, após uma consulta, o paciente poderia ligar para o estudante e falar que está sentindo outros sintomas ou outras situações de risco, a fim de testar as habilidades do aluno em diversas ocasiões. Pretende-se inserir também novas formas de interação entre jogadores, como emoticons e gestos.

A fim de deixar o ambiente mais realista e divertido, espera-se incorporar realidade aumentada no ambiente virtual. A realidade aumentada pode ser utilizada para mostrar instrumentos cirúrgicos para os alunos, utilizados pelos médicos em sala de cirurgias, através de seus celulares. Outra ideia é a de simular consultas no mundo real através da tecnologia, possibilitando que o aluno de medicina consulte um paciente projetado, através de realidade aumentada, no sofá de sua casa.

\section{Referências}

Bez, M. R.; Fonseca, J.; Maroni, V.; Barros, P.; Cazella, S. e Flores, C. D. (2014) "Simulador inteligente para a tomada de decisão em cuidados de saúde - Simdecs". Revista Espaço para a Saúde, v. 15, p. 599-609.

Brasil, I., Neto, F. e Lima, R.; (2011) "Um Jogo Persistente Baseado em Navegador para Treinamento de Operadores de Sondas de Perfuração de Poços de Petróleo". In: XXII Simpósio Brasileiro de Informática na Educação - XVII WIE - Aracaju, SE Brasil.

Cremesp. "Teste reprova 59\% dos estudantes em medicina. 2014." Disponível em: http://www.cremesp.org.br/?siteAcao=Imprensa\&acao=crm_midia\&id=702. Acessado em 01 de Setembro de 2015.

Frade, R.; Neto, F. M. M.,; Lima, R. W.;Lima, R. M. e Silva, L. (2014) “Um Ambiente Virtual 3D Multiagente com Recomendação Personalizada de Objetos de Aprendizagem.” In: III Congresso Brasileiro de Informática na Educação, Dourados. Anais do CBIE, UFGD.

G1. (2012) "Registro de erros médicos crescem no Brasil." Disponível em: http://g1.globo.com/sp/campinas regiao/noticia/2012/05/registros-de-erros-medicoscrescem-52-entre-os-anos-de-2010-e 2011.html. Acessado em 01 de Setembro de 2015 . 


\section{CBIE-LACLO 2015}

Anais dos Workshops do IV Congresso Brasileiro de Informática na Educação (CBIE 2015)

Hamari, J.; Koivisto, J. e Sarsa, H. (2014). "Does gamification work?". In: Hawaii International Conference on System Science. Pág. 3025-3032

Jennifer, G. e Andrew, S., (2010) "Use a Cabeça C\#”. Editora O'Reilly. ISBN: 9788576085591. $2^{\circ}$ Edição.

Jovanovic, J. e Devedzic, V. (2014). Open badges: Challenges and oppor- tunities. In Advances in Web-Based Learning-ICWL 2014, pages 56-65. Springer.

Kera, M.; Pedrini, H. e Nunes, F. (2011) "Ambiente Virtual Interativo com Colisão e Deformação de Objetos para Treinamento Médico". Revista de Informática Teórica e Aplicada - RITA, v.18, pág. 206-233.

Lima, R.; Neto, M.; Almeida, R. (2012) "Um Jogo Sério em 3D para Apoiar a Aprendizagem Sensível ao Contexto dos Estudantes". Congresso Brasileiro de Informática na Educação, Rio de Janeiro. Anais dos Workshops do CBIE 2012 Workshop Ambientes Imersivos para Educação.

Marcial, J. (2014) "Homens Preferem Jogar com Personagens Femininos em World of Warcraft". Disponível em: http://www.diariojovem.com, Acessado em 01 de Setembro de 2015.

Mozilla (2013). Expanding education and workforce opportunities through digital badges. Alliance for Excellent Education - MOZILLA.

Roosendaal, T. (2002) “Blender”. Disponível em: http://www.blender.org, Acessado em 01 de Setembro de 2015.

Silva, S. M. (2010) “JavaScript Guia do Programador”. $1^{\circ}$ Edição, pág. 20-30. Editora Novatec.

Sims, Z. (2015) “Codeacademy”. Disponível em: http://www.codeacademy.com, Acessado em 01 de Setembro de 2015.

Unity (2015) "Development engine for the creation of 2D and 3D games and interactive content". Disponível em: http://unity3d.com/. Acessado em: 01 de Setembro de 2015.

Veras, J.; Labidi, S.; Costa, N. e Pinheiro, T. (2013) "Development of an inteligente virtual environment applied to mastology for diagnosis and training". In: International Conference on Computer Medical Applications (ICCMA). 